\title{
Broadband Amplified Spontaneous Emission and Random Lasing from Wurtzite CdSe/CdS "Giant-Shell" Nanocrystals
}

\author{
Francesco Di Stasio, ${ }^{\dagger, \perp}$ Anatolii Polovitsyn, ${ }^{\dagger, \dagger, \perp}$ Ilaria Angeloni, ${ }^{\dagger, \S}$ Iwan Moreels, ${ }^{\dagger}$ and Roman Krahne ${ }^{*, \dagger}$ \\ ${ }^{\dagger}$ Nanochemistry Department, Istituto Italiano di Tecnologia, Via Morego 30, Genoa 16163, Italy \\ ${ }^{\ddagger}$ Dipartimento di Fisica and ${ }^{\S}$ Dipartimento di Chimica e Chimica Industriale, Università degli Studi di Genova, Via Dodecaneso 33, \\ Genoa 16146, Italy
}

\section{Supporting Information}

ABSTRACT: Colloidal nanocrystals (NCs) are attractive materials for light-emitting applications thanks to their flexible synthesis, size-dependent properties, and bright emission. Yet, colloidal NCs still present a narrow gain band (full-width halfmaximum around $10 \mathrm{~nm}$ ), which limits their application to single-color lasers. Widening of the gain band by specifically engineered NCs can further improve the prospect of this class of materials toward the fabrication of solution-processed white-emitting or color-tunable lasers. Here, we report broadband amplified spontaneous emission (ASE) from wurtzite CdSe/CdS "giant-shell” nanocrystals (g-NCs) with
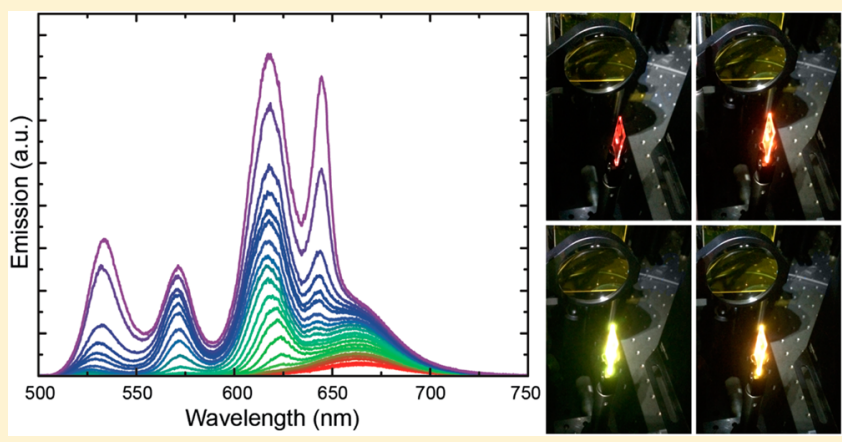
an unprecedented large core up to $7.5 \mathrm{~nm}$ in diameter that were synthesized through a continuous injection route. The combination of large core and shell enables ASE from different CdSe optical transitions as well as from the CdS. Importantly, thin films of g-NCs with a large CdSe core (7.5 and $5.1 \mathrm{~nm}$ in diameter) show ASE at different colors with a similar threshold, indicating that light emission amplification can be achieved from different optical transitions simultaneously. Tuning of the core diameter allows obtaining ASE in a wide spectral range, and blending of gNCs with different core sizes gives rise to a continuous amplified spontaneous emission band from green to red (510 to $650 \mathrm{~nm})$. Drop-cast films of $\mathrm{CdSe} / \mathrm{CdS}$ g-NCs demonstrate simultaneous dual-color random lasing under nanosecond-pulsed excitation.

KEYWORDS: giant-shell nanocrystals, CdSe/CdS, broadband, amplified spontaneous emission, random lasing

$\mathrm{D}$ evelopment of novel semiconductor gain materials for lasers has seen a conspicuous increase in research effort in recent years. A variety of semiconductors able to sustain optical gain in the visible range are available, with colloidal nanocrystals $^{1-6}$ (NCs) and 2D materials ${ }^{7,8}$ being among the most actively investigated. Currently, within the family of solutionprocessed materials, colloidal $\mathrm{CdSe} / \mathrm{CdS}^{2,9-11}$ and perovskite $\mathrm{NCs}^{12-14}$ demonstrate the lowest stimulated emission threshold in the visible spectrum. Colloidal semiconductor NCs based on $\mathrm{CdSe} / \mathrm{CdS}$ core-shell structures have been extensively studied as gain material since the demonstration of stimulated emission, 9 while the development of inorganic ${ }^{3}$ and hybrid ${ }^{12}$ perovskite NCs for lasing applications has just started. Amplified spontaneous emission (ASE) and stimulated emission thresholds as low as a few $\mu \mathrm{J} / \mathrm{cm}^{2}$ have been reported $^{2,6,15}$ for CdSe/CdS core-shell NCs due to optimized synthesis and the reduction of Auger recombination by interfacial engineering ${ }^{6,16}$ and shape control. ${ }^{2,17,18}$ Typically, colloidal NCs present a relatively narrow optical gain band positioned at the band-edge photoluminescence (PL) peak (full-width half-maximum, fwhm, around $10 \mathrm{~nm}$ ). ${ }^{1,3,19}$ Broadening of the optical gain band can pave the way to solutionprocessed wavelength-tunable lasers or white-emitting lasers, which will remove the necessity of using NCs of different composition or sizes ${ }^{19}$ or blends of different semiconducting conjugated polymers for such applications. ${ }^{20}$ Specific architectures such as $\mathrm{CdSe} / \mathrm{CdS}$ core-shell dot-in-rods (DiRs) ${ }^{21-23}$ and giant-shell NCs (g-NCs) $)^{24-26}$ can show ASE from the $\mathrm{CdSe}$ and the $\mathrm{CdS}$ components depending on the core/shell volume ratio and the overall size of the shell, ${ }^{21,22}$ thus enabling optical gain in the green and red part of the visible spectrum. Importantly, g-NCs present a further advantage over DiRs, since they demonstrate gain from two different CdSe core transitions in addition to the shell, as reported by F. GarciaSantamaria et al. ${ }^{27}$ This phenomenon has been observed for gNCs with a relatively small (3 $\mathrm{nm}$ in diameter) zincblende CdSe core, ${ }^{27}$ and a question arises if additional ASE peaks can be intentionally added by using larger cores exploiting higher energy levels or optical transitions. Furthermore, use of a large core will reduce the Auger rates, ${ }^{24,28}$ since the latter is proportional to $1 / r^{3}$ (where $r$ is the core radius).

For these reasons we have investigated a series of g-NCs with increasing core diameter. g-NCs obtained via a continuous injection route can be synthesized starting from wurtzite (WZ) $\mathrm{CdSe}$ cores, leading to a nearly defect-free epitaxial growth of a

Received: June 30, 2016

Published: October 5, 2016 
CdS WZ shell at relatively high reaction temperatures. ${ }^{26} \mathrm{We}$ expanded this synthetic approach to cores with a diameter up to $7.5 \mathrm{~nm}$. Employing a large CdSe core enables further broadening of the ASE band; more specifically four distinct peaks can be observed. Varying the CdSe core diameter, while keeping an overall similar size among the different g-NCs samples allowed for fabrication of solid films consisting of a homogeneous blend leading to a continuous ASE band from 510 to $650 \mathrm{~nm}$. Drop-cast g-NCs films show efficient random lasing from areas where cracks induce light scattering., Importantly, the random lasing is observed at two different colors simultaneously and with similar intensity.

The g-NCs here investigated were synthesized using a continuous injection approach ${ }^{26}$ employing CdSe wurtzite cores previously prepared following the procedure in ref 17 . To obtain isotropic CdSe cores with a $7.5 \mathrm{~nm}$ diameter, the synthesis was slightly modified since the procedure of Carbone et al. $^{17}$ yielded elongated CdSe cores when extending the reaction time. ${ }^{29}$ This anisotropic growth is likely caused by impurities in the trioctylphosphine oxide (TOPO) and octadecylphosphonic acid (ODPA) in the form of shorter phosphonic acids. ${ }^{29,30}$ Slightly reducing the concentration of ODPA, replacing it by an equivalent amount of tetradecylphosphonic acid (TDPA), on the other hand, allowed for the growth of isotropic CdSe cores with a diameter greater than 5.5 $\mathrm{nm}$. In Figure SI1 we report the optical absorption spectra, photoluminescence spectra, transmission electron microscopy (TEM) images, and size distribution histograms of the five $\mathrm{CdSe}$ cores used in our study. Their diameter was determined via the optical absorption spectra, yielding 3.7, 4.1, 4.6, 5.1, and $7.5 \mathrm{~nm} .{ }^{31}$ Good agreement is found with a size analysis via TEM (Figure SI1); for simplicity we will refer to the g-NCs using the sizes obtained through the optical absorption spectra. $^{31}$ Figure $1 \mathrm{a}$ and $\mathrm{b}$ show TEM images of two of the core/shell g-NCs samples used in this study with CdSe core diameters of 3.7 and $7.5 \mathrm{~nm}$, respectively, drop-casted on carbon-coated $\mathrm{Cu}$ grids (TEM images from g-NCs with other core diameters are reported in Figure SI2 in the Supporting Information). All samples are slightly polydisperse, with an
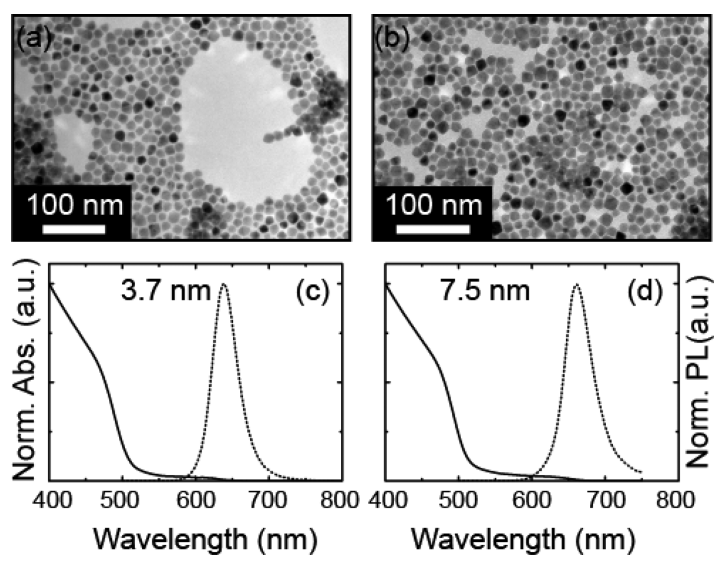

Figure 1. ( $a$ and $b)$ TEM images of the two g-NCs samples: 3.7 and $7.5 \mathrm{~nm}$ core diameters. (c and d) Optical absorption (solid line) and PL spectra (dotted line) of CdSe/CdS g-NCs with $3.7 \mathrm{~nm}$ (c) and 7.5 $\mathrm{nm}$ (d) core diameters. A PL red-shift of $23 \mathrm{~nm}$ is observed when increasing the core size from $3.7 \mathrm{~nm}$ to $7.5 \mathrm{~nm}$. Optical absorption, PL spectra, and TEM images for all other samples are shown in Figure SI1. Optical absorption and PL spectra were collected from dilute gNCs in chloroform. average diameter from 11 to $16 \mathrm{~nm}$ throughout the sample set (see Figure SI3 and Table SI1). The number of CdS monolayers (MLs) forming the shell ranges from 9 to 17 among the g-NCs. Figure 1 shows the PL and optical absorption spectra of the 3.7 and $7.5 \mathrm{~nm}$ g-NCs samples in dilute chloroform solutions (spectra from g-NCs with other core diameters are reported in Figure SI1 in the Supporting Information). Within the g-NC series we observe a PL red-shift of $23 \mathrm{~nm}$ when increasing the core size. All samples present a similar PL fwhm of $44 \pm 3 \mathrm{~nm}$ and a similar absorption spectrum due to the "bulk-like" CdS shell. The CdSe core size has a significant impact on the PL quantum yield (QY) because a larger core diameter will enable a faster shell-to-core carrier relaxation rate due to a decreased shell/core volume ratio. This is confirmed by the range in PLQY from $25 \%$ to $53 \%$ that we measured, where we achieved the highest value for the largest core sample under shell excitation at $450 \mathrm{~nm} .^{26}$

To assess the feasibility of our g-NCs as gain materials, we performed ASE measurements on drop-casted films on sodalime glass slides.

Figure 2 shows the emission spectra recorded from a film of g-NCs with a $7.5 \mathrm{~nm}$ core diameter under femtosecond
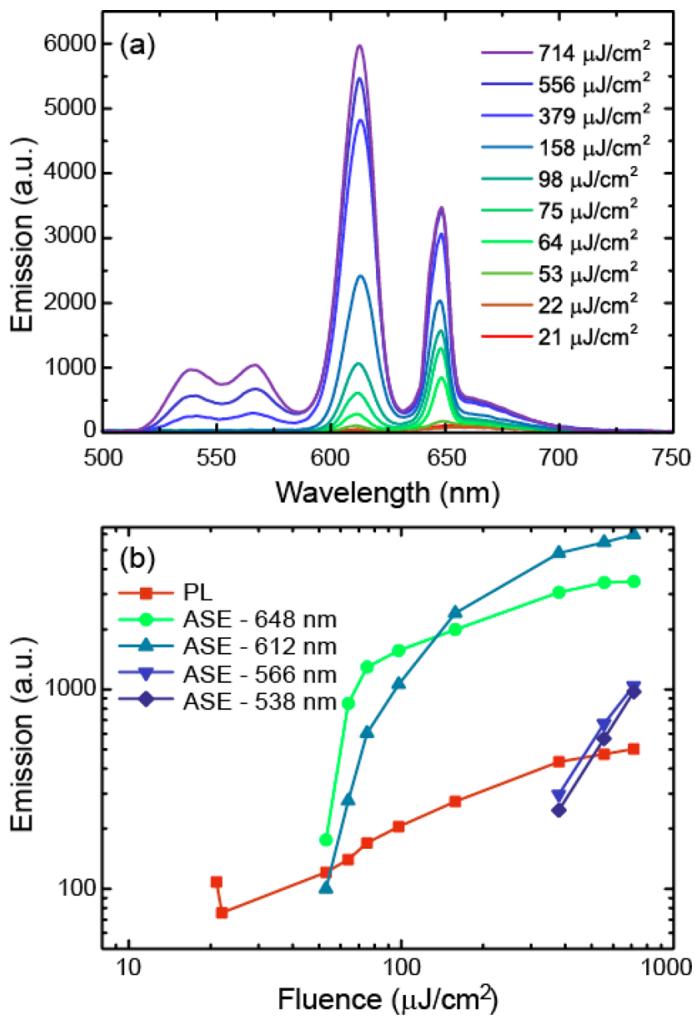

Figure 2. (a) Emission spectra under increasing femtosecond-pulsed excitation fluence with $\lambda_{\text {exc }}=405 \mathrm{~nm}$ recorded from a drop-casted film of CdSe/CdS g-NCs with a $7.5 \mathrm{~nm}$ core diameter. The measurements were carried out using a cylindrical lens to focus the excitation beam into a stripe. ASE from different excited states as well as from the CdS shell is observed. (b) Emission intensity vs excitation fluence curves for the different ASE peaks shown in panel (a).

excitation at increasing pump fluence (the excitation beam was focused in a stripe for all these measurements). ASE is initially observed at 648 and $612 \mathrm{~nm}$ (Figure 2a) for an excitation fluence of $53 \mu \mathrm{J} / \mathrm{cm}^{2}$, close to the PL saturation onset (see Figure $2 \mathrm{~b}$ ). At this point, the question arises if the presence of 
two ASE peaks could be due to sample polydispersity. The CdSe cores have a limited polydispersity of around $10 \%$, but variations in the $\mathrm{CdS}$ shell thickness are clearly observed. Nevertheless, CdS shell thickness variations typically have only a limited impact on the spectral position of the PL (for a shell thickness $\geq 3 \mathrm{~nm}$ ), ruling out sample polydispersity as a possible explanation for the different emission peaks. ${ }^{32,33}$ Therefore, we attribute the ASE peaks to the band-edge emission at $648 \mathrm{~nm}$ and a higher energy optical transition of the g-NC sample. Furthermore, the band-edge ASE band is blueshifted compared to the PL peak, likely due to a repulsive exciton-exciton interaction. ${ }^{5,27,34}$ Such a blue-shift of the bandedge ASE was observed for all core sizes (see Figures S2 and S3). With increasing pump fluence two additional ASE peaks appear at 566 and $538 \mathrm{~nm}$, which can be assigned to a higher order CdSe core energy transition and the CdS shell (at 538 $\mathrm{nm}$, as observed for $\mathrm{CdSe} / \mathrm{CdS}$ DiRs with a large CdS rod length). ${ }^{21,35}$ The appearance of narrow emission peaks (fwhm values are reported in Table 2 of the Supporting Information) already indicates the presence of ASE. To confirm that the observed emission peaks originate from an ASE process, we performed time-resolved measurements (see Figure SI4). The time traces show a shortened temporal decay at high pump fluence for all peaks, which is a clear fingerprint of ASE. ${ }^{1,21}$

We observe a similar behavior to the one reported in Figure 2 for all samples in our series, with ASE from different optical transitions (see Figures SI2 and SI3 in the Supporting Information). The minimum fluence to observe ASE varies from 45 to $105 \mu \mathrm{J} / \mathrm{cm}^{2}$ throughout the sample series, and we find no clear correlation between core size and the onset of ASE, most likely due to the influence of the film morphology on the latter, as reported by C. Gollner et al. ${ }^{5}$

The evolution of the ASE peaks and PL intensities versus excitation fluence for the largest core g-NCs is presented in Figure $2 \mathrm{~b}$, and it shows that the two ASE peaks observed at 648 and $612 \mathrm{~nm}$ occur at similar threshold and the latter presents an overall higher ASE intensity. The $5.1 \mathrm{~nm}$ core g-NCs sample (Figure SI3) also shows a similar ASE threshold for two different optical transitions. Decreasing the core size further results in ASE from an excited-state energy level that appears at higher threshold values compared to that of the band-edge transition. This can be explained by the increased exciton confinement for smaller CdSe cores, which leads to more efficient Auger recombination, thus hindering ASE from higher energy levels. $^{36}$

ASE from an additional (3rd) core transition is clearly observable for the largest core size g-NCs, while in the case of the $5.1 \mathrm{~nm}$ core diameter, this optical gain band is close to the CdS shell (at 546 and $536 \mathrm{~nm}$, respectively). In samples with a smaller core size we observed only three ASE peaks: two from optical transitions of the CdSe core and a third one from the CdS shell.

The observed blue-shift of the ASE peak arising from the band-edge transition (throughout all our samples) suggests that optical gain is obtained through the formation of multiexcitons, similar to what was previously observed for g-NCs. ${ }^{5}$ In order to achieve ASE from multiexcitons, Auger recombination has to be strongly reduced, because Auger rates sharply increase with exciton density, efficiently preventing stimulated emission. ${ }^{6,37}$ We achieved this by the large core size and a large exciton volume in our g-NCs, where holes are strongly confined within the CdSe core and electrons are delocalized. ${ }^{5}$ Importantly, the two largest core samples show two ASE peaks at similar pump fluence, indicating that stimulated emission occurs simultaneously for two different optical transitions, and thus suggesting that the exciton cooling process from higher energy states is slowed down. Depopulation of high-energy states is typically very efficient and takes place within picoseconds. ${ }^{38}$ Considering that cooldown from high-energy levels (associated with the second and third ASE peaks) occurs through the emission of optical phonons of energy around $30 \mathrm{meV}^{38}$ possibly a "phonon bottleneck" 34 prevents efficient energy dissipation, effectively decoupling the different states. This effect, combined with the suppression of Auger recombination for larger cores, can account for the similar threshold of the first and second ASE peaks (Figure $3 b$ ).

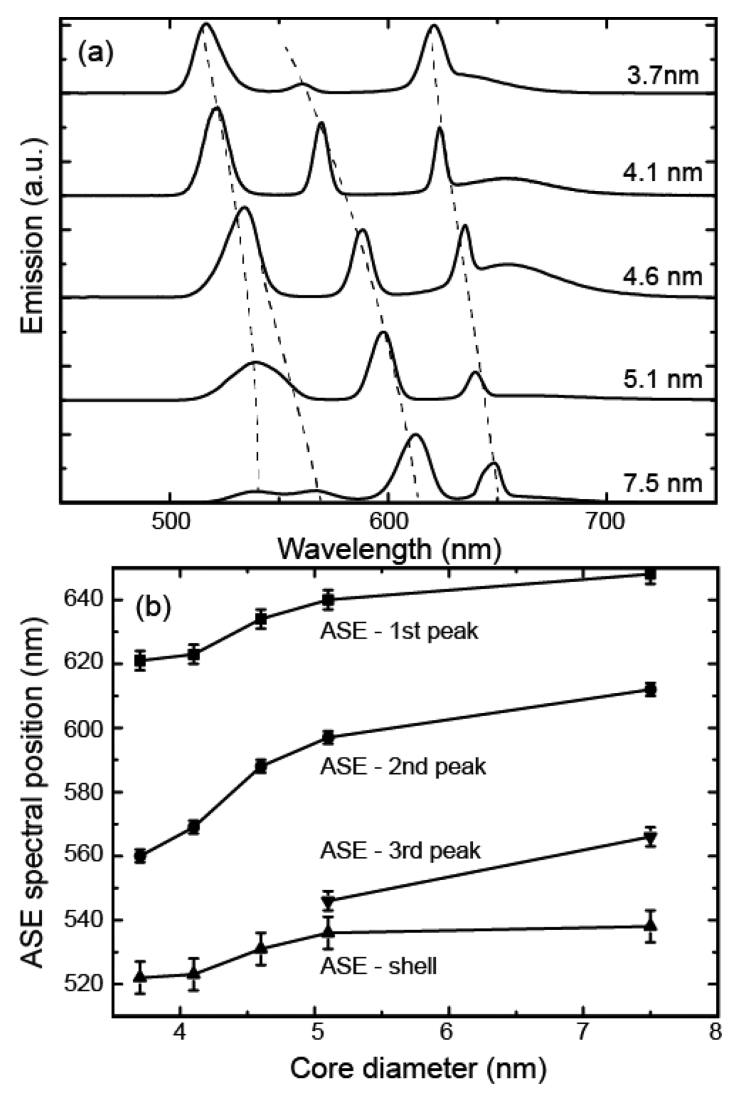

Figure 3. (a) Emission spectra at high excitation fluence for samples with different core size showing ASE from different transitions (dotted lines are guides for the eye). (b) Spectral position of the different ASE peaks vs core sizes (error bars represent the standard deviation).

As discussed above, all g-NC samples show ASE from different $\mathrm{CdSe}$ core emissive states and eventually from the $\mathrm{CdS}$ shell. By changing the core size, it is possible to tune the spectral position of the ASE arising from the different CdSe core transitions. In Figure $3 \mathrm{a}$ emission spectra at high pump fluence of the different g-NCs show a continuous transition toward longer wavelengths for all ASE peaks for increasing $\mathrm{CdSe}$ core diameter, as expected from the reduction in quantum confinement. ${ }^{36}$ Increasing the CdSe core diameter induces a red-shift of the band-edge ASE peak from 621 to 648 $\mathrm{nm}$ (see Figure $3 \mathrm{~b}$ ), while the subsequent gain band undergoes a stronger shift from 560 to $612 \mathrm{~nm}$. ASE arising from a third CdSe optical transition can be clearly observed only for the largest core diameter samples, while for core diameters of 4.6 $\mathrm{nm}$ or smaller we expect this band to overlap the gain band 
observed from the CdS shell. The ASE peak observed from the $\mathrm{CdS}$ shell shows a red-shift with increasing core diameter as well, which could be caused by convolution of emission arising from core and shell states. ${ }^{39}$

In the following we demonstrate that simple blending of gNC solution with different core diameter, and consequently different positions of ASE peaks, can lead to the formation of a continuous optical gain band from red to green. We prepared films via drop-casting of a mixed solution of g-NCs in chloroform and show the emission spectra under femtosecond excitation with increasing pump fluence of a typical blend film (here core diameters were 7.5 and $4.1 \mathrm{~nm}$ ) in Figure 4, where a continuous optical band from 510 to $650 \mathrm{~nm}$ is created.

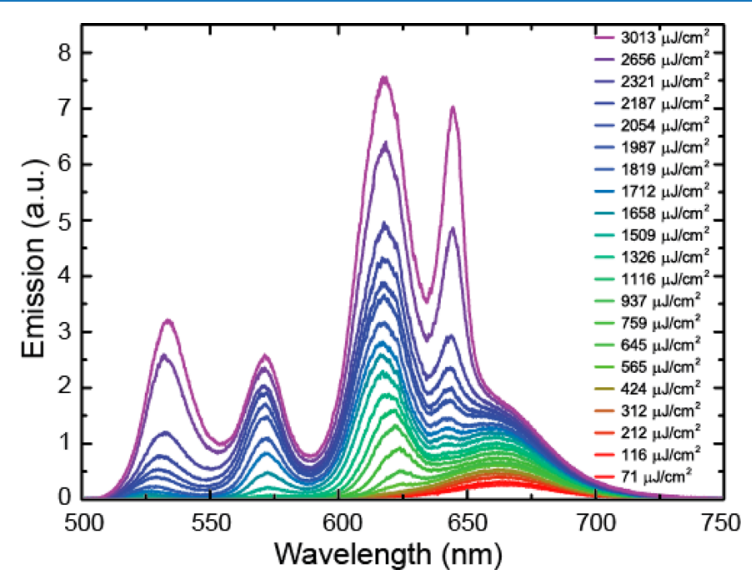

Figure 4. ASE from a blended film of 4.1 and $7.5 \mathrm{~nm}$ core diameter $\mathrm{CdSe} / \mathrm{CdS}$ g-NCs obtained by drop-casting, where broadband optical gain from 510 to $650 \mathrm{~nm}$ is observed.

In the blends, the threshold to obtain ASE is higher than for films from the two separated g-NCs components, which might be correlated to the density of g-NCs of one core size in the excitation spot. ASE is initially observed at $624 \mathrm{~nm}$, which corresponds to the band-edge ASE for the $4.1 \mathrm{~nm}$ core sample (see Figure SI3). On increasing the pump fluence, we observe the appearance of two other peaks, at $643 \mathrm{~nm}$ (band-edge ASE of the $7.5 \mathrm{~nm}$ core sample) and $572 \mathrm{~nm}$ (close to the second ASE peak observed for the $4.1 \mathrm{~nm}$ core sample). Finally, a continuous single band is obtained when the pump fluence is raised above $1.8 \mathrm{~mJ} / \mathrm{cm}^{2}$. To assess the homogeneity of our blended film, we performed confocal imaging, recording the emission in narrow spectral bands centered at wavelengths that correspond to the two different PL peaks of the 4.1 and $7.5 \mathrm{~nm}$ g-NCs (657 and $662 \mathrm{~nm}$, respectively). We did not observe significant differences in intensity in the spatial maps obtained in both spectral bands, which indicates good blending of the two phases. Likely, this was achieved due to the similar overall size of the two g-NC samples despite their different core diameters.

Figure 5a shows an optical microscope image of a film obtained by drop-casting of a solution of $7.5 \mathrm{~nm}$ g-NCs on glass, where a pattern of cracks that formed during solvent evaporation can clearly be observed. The cracks propagate for several micrometers in roughly orthogonal directions in certain areas of the film, forming large aggregates of g-NCs with areas up to $100 \mu \mathrm{m}^{2}$. In contrast to typical random lasing structures ${ }^{40}$ that require an additional scattering medium, such a morphology can induce light scattering and optical feedback due to the relatively high refractive index of CdSe/CdS NCs $(n$
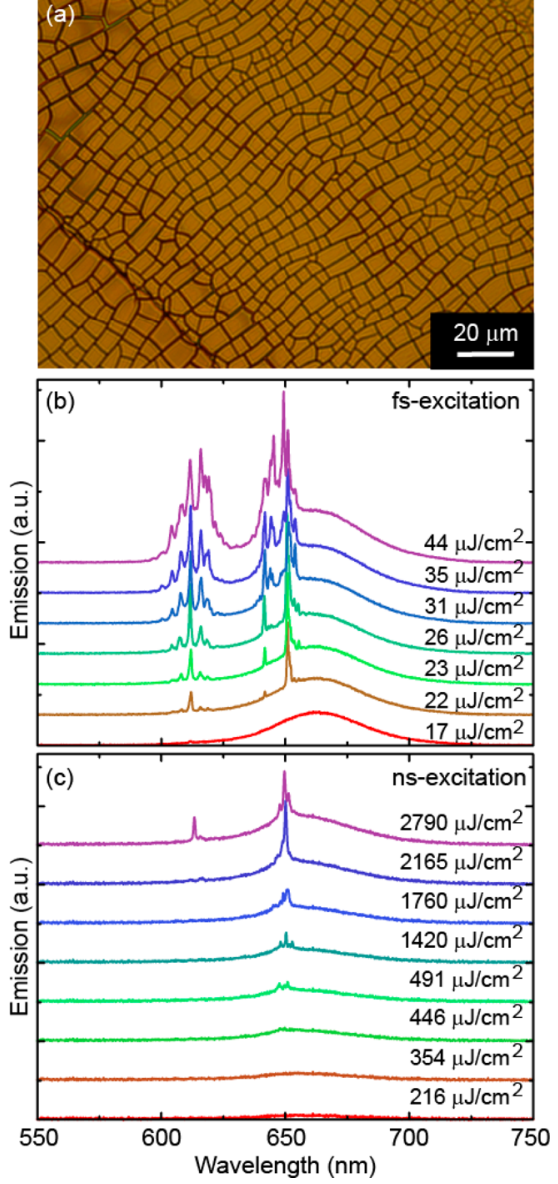

Figure 5. (a) Microscope image of an area with high density of cracks of the film of g-NCs with a $7.5 \mathrm{~nm}$ core diameter. The random lasing arises in particular areas of the drop-cast films where a suitable pattern of cracks is present. (b, c) Emission spectra showing simultaneous random lasing under femtosecond (b) and nanosecond (c) pulsed excitation from band-edge and higher energy core transitions. Here the excitation spot radius was around $600 \mu \mathrm{m}$, and its location was different for the measurements reported in panels (b) and (c).

$\approx 2)^{1}$ with respect to air. For example, coffee rings consisting of $\mathrm{CdSe} / \mathrm{CdS} \mathrm{NCs}$ can act as microresonators and show lasing. ${ }^{1,22,35}$

Figure $5 \mathrm{~b}$ shows efficient dual-color random lasing of a g-NC film from both the band-edge and a higher-energy core transition. Under femtosecond-pulsed excitation, two lasing peaks at 612 and $651 \mathrm{~nm}$ can be observed (fwhm 1.3 and 1.0 $\mathrm{nm}$, respectively) that emerge at a pump fluence of $22 \mu \mathrm{J} / \mathrm{cm}^{2}$. At high pump fluence $\left(31 \mu \mathrm{J} / \mathrm{cm}^{2}\right.$ and above $)$ the spectra show sets of narrow peaks with an envelope that resembles the shape of the ASE bands. We can evaluate the average mode spacing $(\Delta \lambda)$ in each band and obtain $2.3 \mathrm{~nm}$ at the band edge and 3.5 $\mathrm{nm}$ for the higher energy band. From this mode spacing we can estimate the respective cavity round-trip length $\left(s=\lambda^{2} /(n \Delta \lambda)\right)$ as 92 and $53 \mu \mathrm{m}$. ${ }^{6}$ This indicates that the laser modes for the two different transitions originate from different random cavity paths within the film. Importantly, under femtosecond-pulsed excitation we observe lasing at two colors with the same, low pump threshold $\left(22 \mu \mathrm{J} / \mathrm{cm}^{2}\right)$ similar to the ASE behavior (see Figure 2). Measurements performed with nanosecond-pulsed excitation show a lasing threshold around $490 \mu \mathrm{J} / \mathrm{cm}^{2}$ at the band edge $(651 \mathrm{~nm})$, and a set of peaks can be clearly resolved at higher pump fluence. Above $1420 \mu \mathrm{J} / \mathrm{cm}^{2}$ we observe three 
distinct peaks with an average fwhm of $0.6 \mathrm{~nm}$ (close to the resolution limit of our spectrometer) and mode spacing $\Delta \lambda$ around $2.3 \mathrm{~nm}$. On increasing the excitation to $2790 \mu \mathrm{J} / \mathrm{cm}^{2}$, lasing corresponding to the second ASE band is observed in the form of an additional peak at $613 \mathrm{~nm}$ with a fwhm of $1.0 \mathrm{~nm}$ (see Figure 5c). We note that absolute values for lasing threshold between femtosecond and nanosecond excitation are difficult to compare because the threshold depends on local film morphology, and the spectra were not recorded from exactly the same area. Under nanosecond excitation lasing from the higher energy band emerges only at high pump fluence, indicating that on this time scale Auger recombination and electron cooling are more dominant than under femtosecond excitation.

In conclusion, we have developed a modified continuous injection route ${ }^{26}$ that allowed us to synthesize WZ/WZ g-NCs with a core diameter up to $7.5 \mathrm{~nm}$. Such large-core g-NCs are able to sustain ASE from four different optical transitions. Therefore, g-NCs with large CdSe cores have a clear advantage compared to other core-shell architectures, since they demonstrate simultaneous ASE at different colors (i.e., with similar threshold and intensity). Blending of two g-NC samples leads to the formation of a homogeneous solid-state film due to the similar overall sizes of the NCs despite the different core diameters. Such films show a continuous ASE band from 510 to $650 \mathrm{~nm}$, thus paving the way to solution-processed gain materials that can be used for the fabrication of white or wavelength-tunable lasers. Another demonstration of the potential of large core g-NCs for laser applications is the presence of simultaneous dual-color lasing of similar intensity obtained from drop-casted films presenting cracks and inhomogeneity. Such systems present dual-color random lasing even under nanosecond-pulsed excitation.

\section{EXPERIMENTAL SECTION}

Chemicals. Trioctylphosphine oxide (99\%), trioctylphosphine (97\%), sulfur (99\%), and selenium (99,99\%) were purchased from Strem Chemicals. Octadecylphosphonic acid (99\%) and tetradecylphosphonic acid (99\%) were purchased from Polycarbon Industries. Cadmium oxide (99.5\%), 1-octadecene (90\%), and oleic acid (90\%) were purchased from Sigma-Aldrich.

Sample Preparation. The CdSe cores of different diameters were synthesized at $380{ }^{\circ} \mathrm{C}$ following the procedure reported in ref 17 . To obtain CdSe cores with a $7.5 \mathrm{~nm}$ diameter, we used a mixture of TDPA and ODPA in a 15:85 mass ratio instead of pure ODPA. Then a CdS shell was grown as in in ref 26, and the obtained CdSe/CdS gNCs were dispersed in chloroform. Films used for the ASE and lasing measurements were prepared via drop-casting of the chloroform g-NC solution onto previously cleaned soda-lime glass slides under air. The same film preparation was used for the blended film where a solution of mixed 4.1 and $7.5 \mathrm{~nm}$ core diameter g-NCs was employed.

Sample Characterization. Optical absorption spectra were collected from dilute chloroform solutions with a Cary 5000 UVvis-NIR spectrophotometer from Agilent Technologies, while the steady-state PL and PLQY were measured with an Edinburgh Instruments fluorescence spectrometer (FLS920) equipped with a calibrated integrating sphere. g-NC solutions for PLQY measurements were prepared in quartz cuvettes and carefully diluted to 0.1 optical density at the excitation wavelength $(\lambda=450 \mathrm{~nm})$. TEM images were acquired using a JEOL-1100 TEM operating at an acceleration voltage of $100 \mathrm{kV}$.

ASE and Laser Emission Measurements. Films of CdSe/CdS gNCs were excited with $\lambda=405 \mathrm{~nm}$ using an amplified Ti:sapphire laser (Coherent Legend Elite seeded by a Ti:sapphire fs laser) with a $70 \mathrm{fs}$ pulse $(\mathrm{fwhm})$ and a repetition rate of $1 \mathrm{kHz}$. The ASE measurements were performed by focusing the excitation beam with a cylindrical lens onto the sample, thus obtaining a stripe-shaped beam profile. All ASE spectra were collected at $\sim 90^{\circ}$ with respect to the excitation beam. Laser emission measurements on the drop-casted films were carried out using a spherical lens. For nanosecond-pulsed excitation a SpectraPhysics Explorer laser diode $\left(\lambda_{\text {exc }}=349 \mathrm{~nm}, 3 \mathrm{~ns}\right.$ pulse fwhm, repetition rate $1 \mathrm{kHz}$ ) was used. All steady-state emission spectra from films were collected with an Ocean Optics HR4000 spectrometer coupled to an optical fiber. For time-resolved emission spectra below and above threshold a Hamamatsu Photonics streak camera was employed. Emission intensities used in the intensity versus excitation fluence curves (Figure 2b, Figures SI2 and SI3) were obtained via a multipeak fit with two Gaussians to fit the ASE and PL peaks.

Optical Microscope Image. The optical microscope image reported in Figure 5a was acquired in reflection mode using a Nikon microscope with a $60 \times$ objective.

\section{ASSOCIATED CONTENT}

\section{Supporting Information}

The Supporting Information is available free of charge on the ACS Publications website at DOI: 10.1021/acsphotonics.6b00452.

TEM images and light emission data from all samples of the series (PDF)

\section{AUTHOR INFORMATION}

\section{Corresponding Author}

*E-mail: roman.krahne@iit.it.

\section{Author Contributions}

${ }^{\perp}$ F. Di Stasio and A. Polovitsyn contributed equally.

\section{Notes}

The authors declare no competing financial interest.

\section{ACKNOWLEDGMENTS}

The research leading to these results has received funding from the CARIPLO Foundation through the project "NANOCRYSLAS" and from the Ministero degli Affari Esteri e della Cooperazione Internazionale (IONX-NC4SOL). We thank Marco Scotto for valuable technical assistance.

\section{REFERENCES}

(1) Di Stasio, F.; Grim, J. Q.; Lesnyak, V.; Rastogi, P.; Manna, L.; Moreels, I.; Krahne, R. Single-mode lasing from colloidal water-soluble Cdse/CdS quantum dot-in-rods. Small 2015, 11, 1328-1334.

(2) Grim, J. Q.; Christodoulou, S.; Di Stasio, F.; Krahne, R.; Cingolani, R.; Manna, L.; Moreels, I. Continuous-wave biexciton lasing at room temperature using solution-processed quantum wells. Nat. Nanotechnol. 2014, 9, 891-895.

(3) Yakunin, S.; Protesescu, L.; Krieg, F.; Bodnarchuk, M. I.; Nedelcu, G.; Humer, M.; De Luca, G.; Fiebig, M.; Heiss, W.; Kovalenko, M. V. Low-threshold amplified spontaneous emission and lasing from colloidal nanocrystals of caesium lead halide perovskites. Nat. Commun. 2015, 6.805610.1038/ncomms9056

(4) Xu, Y.; Chen, Q.; Zhang, C.; Wang, R.; Wu, H.; Zhang, X.; Xing, G.; Yu, W. W.; Wang, X.; Zhang, Y.; Xiao, M. Two-photon-pumped perovskite semiconductor nanocrystal lasers. J. Am. Chem. Soc. 2016, 138, 3761-3768.

(5) Gollner, C.; Ziegler, J.; Protesescu, L.; Dirin, D. N.; Lechner, R. T.; Fritz-Popovski, G.; Sytnyk, M.; Yakunin, S.; Rotter, S.; Yousefi Amin, A. A.; Vidal, C.; Hrelescu, C.; Klar, T. A.; Kovalenko, M. V.; Heiss, W. Random lasing with systematic threshold behavior in films of $\mathrm{CdSe} / \mathrm{CdS}$ core/thick-shell colloidal quantum dots. ACS Nano 2015, 9, 9792-9801.

(6) Park, Y.-S.; Bae, W. K.; Baker, T.; Lim, J.; Klimov, V. I. Effect of Auger recombination on lasing in heterostructured quantum dots with engineered core/shell interfaces. Nano Lett. 2015, 15, 7319-7328. 
(7) Fan, F.; Turkdogan, S.; Liu, Z.; Shelhammer, D.; Ning, C. Z. A monolithic white laser. Nat. Nanotechnol. 2015, 10, 796-803.

(8) Wu, S.; Buckley, S.; Schaibley, J. R.; Feng, L.; Yan, J.; Mandrus, D. G.; Hatami, F.; Yao, W.; Vuckovic, J.; Majumdar, A.; Xu, X. Monolayer semiconductor nanocavity lasers with ultralow thresholds. Nature 2015, 520, 69-72.

(9) Klimov, V. I.; Mikhailovsky, A. A.; Xu, S.; Malko, A.; Hollingsworth, J. A.; Leatherdale, C. A.; Eisler, H.-J.; Bawendi, M. G. Optical gain and stimulated emission in nanocrystal quantum dots. Science 2000, 290, 314-317.

(10) Alivisatos, A. P. Semiconductor clusters, nanocrystals, and quantum dots. Science 1996, 271, 933-937.

(11) Iyer, V. H.; Mahadevu, R.; Pandey, A. Low threshold quantum dot lasers. J. Phys. Chem. Lett. 2016, 7, 1244-1248.

(12) Vybornyi, O.; Yakunin, S.; Kovalenko, M. V. Polar-solvent-free colloidal synthesis of highly luminescent alkylammonium lead halide perovskite nanocrystals. Nanoscale 2016, 8, 6278-6283.

(13) Protesescu, L.; Yakunin, S.; Bodnarchuk, M. I.; Krieg, F.; Caputo, R.; Hendon, C. H.; Yang, R. X.; Walsh, A.; Kovalenko, M. V. Nanocrystals of cesium lead halide perovskites $(\mathrm{CsPbX} 3, \mathrm{X}=\mathrm{Cl}, \mathrm{Br}$, and I): Novel optoelectronic materials showing bright emission with wide color gamut. Nano Lett. 2015, 15, 3692-3696.

(14) Dou, L.; Wong, A. B.; Yu, Y.; Lai, M.; Kornienko, N.; Eaton, S. W.; Fu, A.; Bischak, C. G.; Ma, J.; Ding, T.; Ginsberg, N. S.; Wang, L.W.; Alivisatos, A. P.; Yang, P. Atomically thin two-dimensional organic-inorganic hybrid perovskites. Science 2015, 349, 1518-1521.

(15) She, C.; Fedin, I.; Dolzhnikov, D. S.; Dahlberg, P. D.; Engel, G. S.; Schaller, R. D.; Talapin, D. V. Red, yellow, green, and blue amplified spontaneous emission and lasing using colloidal CdSe nanoplatelets. ACS Nano 2015, 9, 9475-9485.

(16) Cragg, G. E.; Efros, A. L. Suppression of Auger processes in confined structures. Nano Lett. 2010, 10, 313-317.

(17) Carbone, L.; Nobile, C.; De Giorgi, M.; Sala, F. D.; Morello, G.; Pompa, P.; Hytch, M.; Snoeck, E.; Fiore, A.; Franchini, I. R.; Nadasan, M.; Silvestre, A. F.; Chiodo, L.; Kudera, S.; Cingolani, R; Krahne, R.; Manna, L. Synthesis and micrometer-scale assembly of colloidal CdSe/ CdS nanorods prepared by a seeded growth approach. Nano Lett. 2007, 7, 2942-2950.

(18) Peng, X.; Manna, L.; Yang, W.; Wickham, J.; Scher, E.; Kadavanich, A.; Alivisatos, A. P. Shape control of CdSe nanocrystals. Nature 2000, 404, 59-61.

(19) Dang, C.; Lee, J.; Breen, C.; Steckel, J. S.; Coe-Sullivan, S.; Nurmikko, A. Red, green and blue lasing enabled by single-exciton gain in colloidal quantum dot films. Nat. Nanotechnol. 2012, 7, 335339.

(20) Brovelli, S.; Virgili, T.; Mroz, M. M.; Sforazzini, G.; Paleari, A.; Anderson, H. L.; Lanzani, G.; Cacialli, F. Ultra-broad optical amplification and two-colour amplified spontaneous emission in binary blends of insulated molecular wires. Adv. Mater. 2010, 22, 3690-3694.

(21) Moreels, I.; Rainò, G.; Gomes, R.; Hens, Z.; Stöferle, T.; Mahrt, R. F. Nearly temperature-independent threshold for amplified spontaneous emission in colloidal $\mathrm{CdSe} / \mathrm{CdS}$ quantum dot-in-rods. Adv. Mater. 2012, 24, OP231-OP235.

(22) Zavelani-Rossi, M.; Lupo, M. G.; Krahne, R.; Manna, L.; Lanzani, G. Lasing in self-assembled microcavities of CdSe/CdS core/ shell colloidal quantum rods. Nanoscale 2010, 2, 931-935.

(23) Coropceanu, I.; Rossinelli, A.; Caram, J. R.; Freyria, F. S.; Bawendi, M. G. Slow-injection growth of seeded CdSe/CdS nanorods with unity fluorescence quantum yield and complete shell to core energy transfer. ACS Nano 2016, 10, 3295-3301.

(24) Chen, Y.; Vela, J.; Htoon, H.; Casson, J. L.; Werder, D. J.; Bussian, D. A.; Klimov, V. I.; Hollingsworth, J. A. Giant" multishell cdse nanocrystal quantum dots with suppressed blinking. J. Am. Chem. Soc. 2008, 130, 5026-5027.

(25) Bae, W. K.; Padilha, L. A.; Park, Y.-S.; McDaniel, H.; Robel, I.; Pietryga, J. M.; Klimov, V. I. Controlled alloying of the core-shell interface in $\mathrm{CdSe} / \mathrm{CdS}$ quantum dots for suppression of Auger recombination. ACS Nano 2013, 7, 3411-3419.
(26) Christodoulou, S.; Vaccaro, G.; Pinchetti, V.; De Donato, F.; Grim, J. Q.; Casu, A.; Genovese, A.; Vicidomini, G.; Diaspro, A.; Brovelli, S.; Manna, L.; Moreels, I. Synthesis of highly luminescent wurtzite $\mathrm{CdSe} / \mathrm{CdS}$ giant-shell nanocrystals using a fast continuous injection route. J. Mater. Chem. C 2014, 2, 3439-3447.

(27) García-Santamaría, F.; Chen, Y.; Vela, J.; Schaller, R. D.; Hollingsworth, J. A.; Klimov, V. I. Suppressed auger recombination in "giant" nanocrystals boosts optical gain performance. Nano Lett. 2009, 9, 3482-3488.

(28) Mangum, B. D.; Sampat, S.; Ghosh, Y.; Hollingsworth, J. A.; Htoon, H.; Malko, A. V. Influence of the core size on biexciton quantum yield of giant $\mathrm{CdSe} / \mathrm{CdS}$ nanocrystals. Nanoscale 2014, 6, $3712-3720$.

(29) Wang, W.; Banerjee, S.; Jia, S.; Steigerwald, M. L.; Herman, I. P. Ligand control of growth, morphology, and capping structure of colloidal CdSe nanorods. Chem. Mater. 2007, 19, 2573-2580.

(30) Wang, F.; Tang, R.; Buhro, W. E. The trouble with topo; identification of adventitious impurities beneficial to the growth of cadmium selenide quantum dots, rods, and wires. Nano Lett. 2008, 8 , $3521-3524$.

(31) Jasieniak, J.; Smith, L.; van Embden, J.; Mulvaney, P.; Califano, M. Re-examination of the size-dependent absorption properties of CdSe quantum dots. J. Phys. Chem. C 2009, 113, 19468-19474.

(32) Segarra, C.; Climente, J. I.; Polovitsyn, A.; Rajadell, F.; Moreels, I.; Planelles, J. Piezoelectric control of the exciton wave function in colloidal CdSe/CdS nanocrystals. J. Phys. Chem. Lett. 2016, 7, 21822188.

(33) Accanto, N.; Masia, F.; Moreels, I.; Hens, Z.; Langbein, W.; Borri, P. Engineering the spin-flip limited exciton dephasing in colloidal CdSe/CdS quantum dots. ACS Nano 2012, 6, 5227-5233.

(34) Kelestemur, Y.; Cihan, A. F.; Guzelturk, B.; Demir, H. V. Typetunable amplified spontaneous emission from core-seeded CdSe/CdS nanorods controlled by exciton-exciton interaction. Nanoscale 2014, 6, 8509-8514.

(35) Zavelani-Rossi, M.; Krahne, R.; Della Valle, G.; Longhi, S.; Franchini, I. R.; Girardo, S.; Scotognella, F.; Pisignano, D.; Manna, L.; Lanzani, G.; Tassone, F. Self-assembled CdSe/CdS nanorod microlasers fabricated from solution by capillary jet deposition. Laser Photon. Rev. 2012, 6, 678-683.

(36) Brus, L. Electronic wave functions in semiconductor clusters: Experiment and theory. J. Phys. Chem. 1986, 90, 2555-2560.

(37) Klimov, V. I. Multicarrier interactions in semiconductor nanocrystals in relation to the phenomena of Auger recombination and carrier multiplication. Annu. Rev. Condens. Matter Phys., Langer, J. S., Ed. 2014, 5, 285-31610.1146/annurev-conmatphys-031113133900.

(38) Pandey, A.; Guyot-Sionnest, P. Slow electron cooling in colloidal quantum dots. Science 2008, 322, 929-932.

(39) Lupo, M. G.; Della Sala, F.; Carbone, L.; Zavelani-Rossi, M.; Fiore, A.; Lüer, L.; Polli, D.; Cingolani, R.; Manna, L.; Lanzani, G. Ultrafast electron-hole dynamics in core/shell CdSe/CdS dot/rod nanocrystals. Nano Lett. 2008, 8, 4582-4587.

(40) Wiersma, D. S. The physics and applications of random lasers. Nat. Phys. 2008, 4, 359-367. 